\title{
The simultaneity in human capital estimations: The case of China
}

\author{
Tam Bang Vu \\ (College of Business and Economics, University of Hawaii-Hilo, Hawaii 96720, USA)
}

\begin{abstract}
Existing papers on human capital and growth in China has been using single equation estimations. This might cause a simultaneity bias if a two-way causality between the two variables exists. In this paper, the author performs vector autoregressive estimations using panel data on the number of graduates at each level of education as a proxy for human capital in China during 1991-2005. The results show that investment in human capital increases output per worker at all three levels of education. Regarding the effects of output per worker on the accumulation of human capital, the author finds mixed results with the primary-school graduates' benefits the most from increases in per capita output.
\end{abstract}

Key words: simultaneous equation estimations; education; productivity; China’s economy

\section{Introduction}

China's economy has been an attractive subject for many researchers. However, most of the papers focus on GDP growth. Concerning human capital and GDP growth, empirical studies have consistently show that human capital has positive effects on GDP growth ${ }^{1}$. On the contrary, the results on human capital and output per worker have been mixed. Hua (2006) uses yearly data for 29 regions in China to investigate the direct effects of human capital on productivity, as evidence by technical efficiency changes, technical progress, and total factor productivity. Using yearly data on numbers of graduates and single equation estimations, he finds that the effects of secondary and primary education on productivity are either negative or insignificant, whereas that of college education is positive and significant. Hua also finds that the combined effect of all three levels of education is only weakly significant.

In contrast to Hua (2006), Fleisher, et al (2006) estimate the effect of human capital on productivity using yearly data at firm level from China. They divide workers into less-educated and highly educated ones. Performing single equation estimations, they show that investment in human capital increases productivity at both levels with higher level of education has stronger effect than lower level of education ${ }^{2}$.

Vu, Im and Shima (2009) use the ratio of school enrollments to population as a proxy for investment in human capital. They follow Barro and Sala-i-Martin (2004) in calculating three-year average values of each variable to eliminate the short run impacts in the economy. Estimating these averaged data using fixed effect methodology, they find that investment in human capital increases productivity at all three levels of education, but the coefficient of primary education is only significant at ten percent level.

Nonetheless, new research points out a possible two-way causality between human capital and output per

Tam Bang Vu, assistant professor, College of Business and Economics, University of Hawaii-Hilo; research fields: Asian economies, macroeconomics, econometrics.

1 See, Fleisher (2002), Demuger (2001), and Chen and Feng (2000).

2 Yunhua, et al (2000) use microeconomic yearly data of 140 industrial township and village enterprises located in 15 counties in Jiangsu province. They find that higher education is associated with higher productivity. 
worker. Kumar (2003) develops a model addressing this possible two-way causality between human capital and productivity for small open economies. Using simultaneous equation estimations on cross sectional data, he finds that human capital fosters productivity grows, but this growth in turn has a negative effect on human capital accumulation. Using simultaneous equation estimations on panel data for all countries, Vu and Hammes (2007a) also find that human capital increases output per worker, and this growth in turn has a positive effect on the accumulation of human capital.

Since all the existing papers on China's education and productivity use single equation estimations, the remaining question is whether a two-way causality exists for the case of China. In this paper, we let human capital and output per worker is determined simultaneously using data on numbers of graduates for each level of education in China during 1991-2005. Data are averaged every three-year period to reduce the business cycle effect. Performing vector autoregressive estimations (VAR), we find that investment in human capital at all three levels of education increases output per worker. Regarding the effects of output per worker on the accumulation of human capital, we find mixed results with the investment in primary education benefit the most from output per worker increases. Section 2 of this paper presents the model and data. Section 3 discusses methodology and results. Section 4 concludes.

\section{Model and data}

We use an augmented Cobb-Douglas production function in log form similar to a new growth model in Romer (2003) or Thirlwall (2003).

$$
\ln y_{i t}=\ln A+\alpha \ln k_{i t}+\theta \ln h_{i t}+\sum_{j=1}^{n} \gamma_{j} \ln c_{j t}
$$

where $y$ is real GDP per worker, $i$ is province index and $t$ time index, $k$ capital per worker, and $h$ human capital; $c$ is a vector of control variables that might affect labor productivity such as GDP per capita, infrastructure, exports, imports, etc.

Since there is no theoretical model for a large economy like China, we use VAR approach as discussed in Pindyck and Rubinfeld (1998) and write the system as:

$$
\begin{gathered}
\Delta \ln y_{i t}=\Delta \alpha \ln y_{i, t-1}+\Delta \chi \ln k_{i, t-1}+\theta \Delta \ln h_{i, t-1}+\sum_{j=1}^{n} \gamma_{j} \Delta \ln c_{j, t-1} \\
\Delta \ln h_{i t}=\eta \Delta \ln h_{i, t-1}+\lambda \Delta \ln y_{i, t-1}+\sum_{p=1}^{r} \kappa_{j} \Delta \ln c_{j, t-1}
\end{gathered}
$$

In contrast to Fleisher, et al (2006), who use number of years in schools, and Vu, Im, and Shima (2009), who divide numbers of school enrollments by numbers of worker, we divide numbers of graduates from each level of education by numbers of workers (henceforth called the graduate ratio) as a proxy for investment in human capital. We believe that this new method gives a better measure of human capital than the measure of school enrollments. The former represents a capital asset to the society while the latter is a flow variable that incurs repeated counts: A grade-school student has to enroll six years at primary or secondary level before graduating, and a college students has to enroll four or five years before graduating.

Data for 29 regions — including 22 provinces, four autonomous regions, and three municipal cities — are from China Statistical Yearbooks for 1991-2005. Data for Tibet are not comprehensive and therefore are eliminated 
from the data set. Data for Chongqing were included in data for Sichuan before 1997, so we add data for Chonging to data for Sichuan during 1997-2005 as well. Data for 1991-1992 have missing observations. If one observation is missing for a three-year period, we average the two remaining data points. This may bias the results. Hence, we also perform estimations on the data for 1994-2005 as a robust check.

We use accumulated investment in fixed assets by region as a proxy for physical capital, turnover volume of freight traffic as a proxy for infrastructure, and total retail sale of consumer goods as a proxy for trade. The ratio of GDP to population is used as a proxy for per capita income. Data on utilized foreign direct investment are merged with data on foreign other investment for 1993-1995. Hence, we sum up the two categories when they are listed separately to obtain data for foreign investment (FI). Data are converted into real values using the price indices and respective GDP deflators. Data on exports (EX) and imports (IM) are measured according to location of managing unit by region. Data on foreign loans are accumulated to make a proxy for foreign capital (FOCAP).

\section{Methodology and results}

A preliminary simultaneous-equation estimation, which uses all available variables that might affect productivity and human capital, confirms our intuition of a possible two-way causality between productivity and human capital. This estimation and the subsequently preliminary estimations are carried out with time dummies to control for autocorrelation. We also employ the White correction for the standard heteroskedasticity.

We follow a downward piece-wise approach to avoid omitted variables, starting with all available variables. We then perform Variance Inflation Factor tests $(V I F)^{3}$ to eliminate variables with high multicolinearity. After several elimination steps, we have the following system of econometric equations:

$$
\begin{aligned}
\text { OUT }_{i t}= & \beta_{o}+\beta_{1} \text { HUM }_{i, t-1}+\beta_{2} \text { CAP }_{i, t-1}+\beta_{3} \text {TRADE}_{i, t-1}+\beta_{4} E X_{i, t-1} \\
& +\beta_{5} \text { OUT }_{i, t-1}+\beta_{6} \text { FI I }_{i, t-1}+\beta_{7} \text { FOCAP }_{, i-1 t}+v_{t}^{\Delta O U T} \\
\text { HUM }_{i t} & =\beta_{o}+\beta_{1} \text { PROD }_{i, t-1}+\beta_{2} \text { PERCA }_{i, t-1}+\beta_{3} \text { POP }_{i, t-1} \\
& +\beta_{4} \text { RATIO }_{i, t-1}+\beta_{4} \text { RATIO }_{i, t-1}+u_{t}^{\Delta H U M}
\end{aligned}
$$

where OUT is output per worker, HUM is human capital, CAP is physical capital per worker (henceforth called capital), TRADE is domestic trade, $E X$ is exports, $P O P$ is population, $F I$ is foreign investment, FOCAP is foreign capital, PERCA is per capita income, RATIO is student-teacher ratio, $i$ and $t$ are the regional and time indices, respectively, all are in log differences as proxies for growth rates. The variable TRADE has VIF varies between 9.89 and 10.76, depending on the sample sizes. Kennedy (2006) suggests that we eliminate any variable with VIF $>10$, hence we also estimate an alternative model specification without TRADE as another robust check.

The results for primary, secondary and college graduate ratios are reported in Table 1a. It reports the effects of investment in human capital and other variables on output per worker as shown in the first equation of system (3). Each level is estimated separately using alternative time periods of 1991-2005 and 1994-2005. From this table, investment in human capital at all three levels increases productivity. The co-efficient estimates of primary and secondary graduate ratios are highly significant for both sample periods.

The signs and the magnitudes of the coefficients for the three levels are similar to each other. They are also

\footnotetext{
${ }^{3}$ For a discussion of the VIF test, please see Kennedy (2003).
} 
similar to the results from Fleisher, et al (2006) using microeconomic data: Human capital increases productivity for both highly educated workers and less educated workers, with the effect on highly educated workers much greater than that on the less educated workers.

Table $1 \mathrm{~b}$ reports the effect of output per worker and other variables on each level of education as shown in the second equation of system (3). Column (1) and Column (2) report the effect on primary education, Column (3) and Column (4) on secondary education, and Column (5) and Column (6) on college education. Intriguingly, we find that the effects are different across levels. The effects are positive and highly significant effect for primary education and secondary education but with the effect on primary education is more than triple that on secondary education. The effect is negative and statistically significant for college education.

Table 1 Two-way causality between output per worker and each level of education: Model with trade Table 1a Dependent variable: Output per worker

\begin{tabular}{|c|c|c|c|c|c|c|}
\hline \multirow{2}{*}{ Variable } & \multicolumn{2}{|c|}{ Primary } & \multicolumn{2}{|c|}{ Secondary } & \multicolumn{2}{|c|}{ College } \\
\hline & 1991-2005 & 1994-2005 & 1991-2005 & 1994-2005 & 1991-2005 & 1994-2005 \\
\hline \multirow{2}{*}{ HUM } & $0.1104^{* *}$ & $0.1131^{* *}$ & $0.1362 * * *$ & $0.1437 * * *$ & $0.0781 * *$ & $0.0832 * *$ \\
\hline & $(0.0527)$ & $(0.0532)$ & $(0.0465)$ & $(0.0553)$ & $(0.0343)$ & $(0.0405)$ \\
\hline \multirow{2}{*}{ FI } & $0.4362 * * *$ & $0.4819 * * *$ & $0.4102 * * *$ & $0.4121 * * *$ & $0.3259 * * *$ & $0.3625^{* *}$ \\
\hline & (0.0469) & $(0.0434)$ & $(0.0564)$ & $(0.0567)$ & $(0.0352)$ & $(0.0435)$ \\
\hline \multirow{2}{*}{ OUTt-1 } & $0.0664 * * *$ & $0.0304 * *$ & $0.0352 * * *$ & $0.0475^{* *}$ & $0.0387 * * *$ & $0.0429 * *$ \\
\hline & $(0.0143)$ & $(0.0124)$ & $(0.0165)$ & $(0.0249)$ & $(0.0101)$ & $(0.0201)$ \\
\hline \multirow{2}{*}{ CAP } & $0.2653 * * *$ & $0.2814 * * *$ & $0.2998 * * *$ & $0.2856 * * *$ & $0.2785 * * *$ & $0.2923^{* * *}$ \\
\hline & $(0.0642)$ & $(0.0489)$ & $(0.0376)$ & $(0.0564)$ & $(0.0476)$ & $(0.0586)$ \\
\hline \multirow{2}{*}{ TRADE } & $0.0327 * *$ & $0.0425^{* *}$ & $0.0397 * * *$ & $0.0463 * *$ & $0.0343 * *$ & $0.0312 * *$ \\
\hline & $(0.0154)$ & $(0.0214)$ & (0.0104) & $(0.0223)$ & $(0.0171)$ & $(0.0159)$ \\
\hline \multirow{2}{*}{ FOCAP } & $0.0325 * *$ & $0.0365 * *$ & $0.0358 * * *$ & $0.0473 * *$ & $0.0308^{* * *}$ & $0.0423 * *$ \\
\hline & $(0.0176)$ & $(0.0181)$ & $(0.0121)$ & $(0.0243)$ & $(0.0110)$ & $(0.0201)$ \\
\hline Overall R-squared & 0.7001 & 0.6885 & 0.7851 & 0.7232 & 0.6971 & 0.6262 \\
\hline Observations & 145 & 116 & 145 & 116 & 116 & 87 \\
\hline
\end{tabular}

Table 1b Dependent variable: Human capital at each level of education

\begin{tabular}{|c|c|c|c|c|c|c|}
\hline \multirow{2}{*}{ Variable } & \multicolumn{2}{|c|}{ Primary } & \multicolumn{2}{|c|}{ Secondary } & \multicolumn{2}{|c|}{ College } \\
\hline & 1991-2005 & 1994-2005 & 1991-2005 & 1994-2005 & $1991-2005$ & 1994-2005 \\
\hline OUT & $\begin{array}{l}0.2243 * * \\
(0.1012)\end{array}$ & $\begin{array}{l}0.2317 * * \\
(0.1114)\end{array}$ & $\begin{array}{l}0.0707 * * \\
(0.0348)\end{array}$ & $\begin{array}{c}0.0715^{*} \\
(0.0362)\end{array}$ & $\begin{array}{l}-0.0489 * * \\
(0.0223)\end{array}$ & $\begin{array}{l}-0.0382 * * \\
(0.0187)\end{array}$ \\
\hline HUMt-1 & $\begin{array}{l}0.0256 * * \\
(0.0170)\end{array}$ & $\begin{array}{l}0.0352 * * \\
(0.0178)\end{array}$ & $\begin{array}{l}0.0583^{* *} \\
(0.0121)\end{array}$ & $\begin{array}{l}0.0736^{* *} \\
(0.0483)\end{array}$ & $\begin{array}{l}0.0889 * * * \\
(0.0190)\end{array}$ & $\begin{array}{l}0.0423^{* *} \\
(0.0209)\end{array}$ \\
\hline RATIO & $\begin{array}{l}-0.2254^{*} \\
(0.1324)\end{array}$ & $\begin{array}{l}-0.2114 * * * \\
(0.0918)\end{array}$ & $\begin{array}{l}-0.2021^{* *} \\
(0.1012)\end{array}$ & $\begin{array}{l}-0.2108^{* *} \\
(0.1032)\end{array}$ & $\begin{array}{l}-0.2312^{* * *} \\
(0.0104)\end{array}$ & $\begin{array}{l}-0.2412^{* *} \\
(0.2154)\end{array}$ \\
\hline PERCA & $\begin{array}{l}0.3278 * * \\
(0.1546)\end{array}$ & $\begin{array}{l}0.4255^{* *} \\
(0.2147)\end{array}$ & $\begin{array}{l}0.3979 * * \\
(0.1984)\end{array}$ & $\begin{array}{l}0.4638^{* *} \\
(0.2238)\end{array}$ & $\begin{array}{l}0.3439 * * \\
(0.1771)\end{array}$ & $\begin{array}{l}0.3127^{* *} \\
(0.1589)\end{array}$ \\
\hline POP & $\begin{array}{l}0.3255^{* *} \\
(0.1706)\end{array}$ & $\begin{array}{l}0.3659 * * \\
(0.1781)\end{array}$ & $\begin{array}{l}0.3583^{* *} \\
(0.1721)\end{array}$ & $\begin{array}{l}0.4736^{* *} \\
(0.2483)\end{array}$ & $\begin{array}{l}0.3088^{* * *} \\
(0.1190)\end{array}$ & $\begin{array}{l}0.4238^{* *} \\
(0.2091)\end{array}$ \\
\hline Overall R-squared & 0.7031 & 0.6845 & 0.6865 & 0.6732 & 0.6978 & 0.6565 \\
\hline $\mathrm{P}$-value for the F-test & 0.0000 & 0.0000 & 0.0000 & 0.0000 & 0.0000 & 0.0000 \\
\hline
\end{tabular}

Notes: The $*, * *$ and $* * *$ denote $10 \%, 5 \%$ and $1 \%$ significance levels, respectively. Standard errors are in the parentheses.

Table 2 reports the results for the model without TRADE and shows that they are very similar to those in Table 1. 
The simultaneity in human capital estimations: The case of China

Table 2 Two-way causality between output per worker and each level of education: Model without trade Table 2a Dependent variable: Output per worker

\begin{tabular}{|c|c|c|c|c|c|c|}
\hline \multirow{2}{*}{ Variable } & \multicolumn{2}{|c|}{ Primary } & \multicolumn{2}{|c|}{ Secondary } & \multicolumn{2}{|c|}{ College } \\
\hline & 1991-2005 & 1994-2005 & 1991-2005 & 1994-2005 & 1991-2005 & 1994-2005 \\
\hline \multirow{2}{*}{ HUM } & $0.1009 * *$ & $0.1037 * *$ & $0.1263 * * *$ & $0.1275^{* * *}$ & $0.0867 * *$ & $0.0832 * *$ \\
\hline & $(0.0513)$ & $(0.0465)$ & $(0.0564)$ & $(0.0234)$ & $(0.0412)$ & $(0.0412)$ \\
\hline \multirow{2}{*}{ FI } & $0.3343 * * *$ & $0.3547 * *$ & $0.3867 * * *$ & $0.4012 * *$ & $0.3867 * * *$ & $0.3978 * * *$ \\
\hline & $(0.1535)$ & $(0.1724)$ & $(0.0561)$ & $(0.2132)$ & $(0.0425)$ & $(0.0523)$ \\
\hline \multirow{2}{*}{$\mathrm{OUT}_{\mathrm{t}-1}$} & $0.0598 * *$ & $0.0476 * *$ & $0.0352 * * *$ & $0.0452 * *$ & $0.0398 * *$ & $0.0422 * *$ \\
\hline & $(0.0301)$ & $(0.0223)$ & $(0.0169)$ & $(0.0221)$ & $(0.0191)$ & $(0.0212)$ \\
\hline \multirow{2}{*}{ CAP } & $0.2812 * * *$ & $0.2954 * * *$ & $0.2902 * * *$ & $0.2874 * * *$ & $0.2859 * * *$ & $0.2972 * *$ \\
\hline & $(0.0698)$ & $(0.0482)$ & $(0.0379)$ & $(0.0562)$ & $(0.0478)$ & (0.1382) \\
\hline \multirow{2}{*}{ TRADE } & $0.0214 * *$ & $0.0302 * *$ & $0.0243^{* * *}$ & $0.0254 * *$ & $0.0215 * *$ & $0.0274 * *$ \\
\hline & $(0.0112)$ & $(0.0164)$ & $(0.0115)$ & $(0.0206)$ & $(0.0114)$ & $(0.0138)$ \\
\hline \multirow{2}{*}{ FOCAP } & $0.0317^{* *}$ & $0.0366 * *$ & $0.0352 * * *$ & $0.0479 * *$ & $0.0302 * * *$ & $0.0424^{* *}$ \\
\hline & (0.0179) & (0.0183) & $(0.0128)$ & $(0.0245)$ & $(0.0117)$ & (0.0201) \\
\hline Overall R-squared & 0.7034 & 0.6893 & 0.7045 & 0.7276 & 0.6897 & 0.6966 \\
\hline \multirow[t]{2}{*}{ Observations } & 145 & 116 & 145 & 116 & 116 & 87 \\
\hline & Table $2 \mathbf{b}$ & endent variabl & Human capita & t each level & ducation & \\
\hline \multirow{2}{*}{ Variable } & \multicolumn{2}{|c|}{ Primary } & \multicolumn{2}{|c|}{ Secondary } & \multicolumn{2}{|c|}{ College } \\
\hline & 1991-2005 & 1994-2005 & 1991-2005 & 1994-2005 & 1991-2005 & 1994-2005 \\
\hline \multirow{2}{*}{ OUT } & $0.2165 * *$ & $0.2212 * *$ & $0.0692 * *$ & $0.0701 *$ & $-0.0482 * *$ & $-0.0389 * *$ \\
\hline & (0.1019) & $(0.1123)$ & $(0.0342)$ & $(0.0369)$ & $(0.0225)$ & $(0.0182)$ \\
\hline \multirow{2}{*}{$\mathrm{HUM}_{\mathrm{t}-1}$} & $0.0289 * *$ & $0.0363 * *$ & $0.0435 * *$ & $0.0731 * *$ & $0.0882 * * *$ & $0.0429 * *$ \\
\hline & $(0.0179)$ & $(0.0172)$ & $(0.0124)$ & $(0.0489)$ & $(0.0193)$ & $(0.0203)$ \\
\hline \multirow{2}{*}{ RATIO } & $-0.2051^{*}$ & $-0.2119 * * *$ & $-0.2024 * *$ & $-0.2103^{* *}$ & $-0.2317 * *$ & $-0.2415^{* *}$ \\
\hline & $(0.1321)$ & $(0.0916)$ & (0.1019) & $(0.1034)$ & $(0.1092)$ & $(0.2151)$ \\
\hline \multirow{2}{*}{ PERCA } & $0.3171^{* *}$ & $0.4256^{* *}$ & $0.3973 * *$ & $0.4631 * *$ & $0.3432 * *$ & $0.3123^{* *}$ \\
\hline & $(0.1541)$ & $(0.2142)$ & $(0.1984)$ & $(0.2238)$ & $(0.1771)$ & $(0.1589)$ \\
\hline \multirow{2}{*}{ POP } & $0.3352 * *$ & $0.3623 * *$ & $0.3588 * *$ & $0.4731 * *$ & $0.3082 * * *$ & $0.4231^{* *}$ \\
\hline & $(0.1702)$ & $(0.1789)$ & $(0.1729)$ & $(0.2482)$ & $(0.1198)$ & $(0.2091)$ \\
\hline Overall R-squared & 0.7176 & 0.6948 & 0.6972 & 0.6943 & 0.7231 & 0.6895 \\
\hline P-value for the F-test & 0.0000 & 0.0000 & 0.0000 & 0.0000 & 0.0000 & 0.0000 \\
\hline
\end{tabular}

Notes: The $*, * *$ and $* * *$ denote $10 \%, 5 \%$ and $1 \%$ significance levels, respectively. Standard errors are in the parenthese.

Table 3 Two-way causality between output per worker and combined level of education: Model with trade Table 3a Dependent variable: Output per worker

\begin{tabular}{|c|c|c|c|c|c|c|}
\hline \multirow{2}{*}{ Variable } & \multicolumn{2}{|c|}{ Primary+ Secondary } & \multicolumn{2}{|c|}{ Secondary+ College } & \multicolumn{2}{|c|}{ All three levels } \\
\hline & 1991-2005 & 1994-2005 & $1991-2005$ & $1994-2005$ & $1991-2005$ & 1994-2005 \\
\hline HUM & $\begin{array}{l}0.2124 * * \\
(0.1324)\end{array}$ & $\begin{array}{l}0.2136^{* * *} \\
(0.0532)\end{array}$ & $\begin{array}{l}0.2295^{* *} \\
(0.1069)\end{array}$ & $\begin{array}{l}0.2321 * * \\
(0.1153)\end{array}$ & $\begin{array}{l}0.2485^{* * *} \\
(0.0343)\end{array}$ & $\begin{array}{l}0.2523 * * * \\
(0.0402)\end{array}$ \\
\hline FI & $\begin{array}{l}0.2362^{* *} \\
(0.1269)\end{array}$ & $\begin{array}{l}0.4145^{* *} \\
(0.1231)\end{array}$ & $\begin{array}{l}0.3725^{* * *} \\
(0.0612)\end{array}$ & $\begin{array}{l}0.4012 * * * \\
(0.0349)\end{array}$ & $\begin{array}{l}0.3232^{* *} \\
(0.1397)\end{array}$ & $\begin{array}{l}0.3614^{* * *} \\
(0.0475)\end{array}$ \\
\hline $\mathrm{OUT}_{\mathrm{t}-1}$ & $\begin{array}{l}0.0476^{* * *} \\
(0.0198)\end{array}$ & $\begin{array}{l}0.0309^{* *} \\
(0.0121)\end{array}$ & $\begin{array}{l}0.0359 * * * \\
(0.0161)\end{array}$ & $\begin{array}{l}0.0478^{* *} \\
(0.0242)\end{array}$ & $\begin{array}{l}0.0389 * * * \\
(0.0112)\end{array}$ & $\begin{array}{l}0.0421^{* *} \\
(0.0221)\end{array}$ \\
\hline CAP & $\begin{array}{l}0.2978 * * * \\
(0.0674)\end{array}$ & $\begin{array}{l}0.2735^{* * *} \\
(0.0486)\end{array}$ & $\begin{array}{l}0.2986^{* * *} \\
(0.0332)\end{array}$ & $\begin{array}{l}0.2874^{* * *} \\
(0.0585)\end{array}$ & $\begin{array}{l}0.2712^{* * *} \\
(0.0465)\end{array}$ & $\begin{array}{l}0.2627^{* * *} \\
(0.0332)\end{array}$ \\
\hline TRADE & $\begin{array}{l}0.0386^{* *} \\
(0.0187)\end{array}$ & $\begin{array}{l}0.0425^{* *} \\
(0.0219)\end{array}$ & $\begin{array}{l}0.0335^{* *} \\
(0.0142)\end{array}$ & $\begin{array}{l}0.0464^{* *} \\
(0.0298)\end{array}$ & $\begin{array}{l}0.0337^{* *} \\
(0.0113)\end{array}$ & $\begin{array}{l}0.0342 * * \\
(0.0143)\end{array}$ \\
\hline FOCAP & $\begin{array}{l}0.0398^{* *} \\
(0.0132)\end{array}$ & $\begin{array}{l}0.0313^{* *} \\
(0.0123)\end{array}$ & $\begin{array}{l}0.0331^{* *} \\
(0.0179)\end{array}$ & $\begin{array}{l}0.0436^{* *} \\
(0.0232)\end{array}$ & $\begin{array}{l}0.0386^{* *} \\
(0.0182)\end{array}$ & $\begin{array}{l}0.0480^{* *} \\
(0.0245)\end{array}$ \\
\hline Overall R-squared & 0.7085 & 0.6813 & 0.7821 & 0.7279 & 0.6915 & 0.6229 \\
\hline Observations & 145 & 116 & 145 & 116 & 116 & 87 \\
\hline
\end{tabular}


The simultaneity in human capital estimations: The case of China

Table 3b Dependent variable: Human capital at combined level of education

\begin{tabular}{|c|c|c|c|c|c|c|}
\hline \multirow{2}{*}{ Variable } & \multicolumn{2}{|c|}{ Primary+ Secondary } & \multicolumn{2}{|c|}{ Secondary+ College } & \multicolumn{2}{|c|}{ All three levels } \\
\hline & 1991-2005 & 1994-2005 & 1991-2005 & 1994-2005 & 1991-2005 & 1994-2005 \\
\hline OUT & $\begin{array}{l}0.2115^{* *} \\
(0.1045)\end{array}$ & $\begin{array}{l}0.2261 * * \\
(0.1102)\end{array}$ & $\begin{array}{l}-0.0728^{* *} \\
(0.0332)\end{array}$ & $\begin{array}{l}-0.0742 * * \\
(0.0313)\end{array}$ & $\begin{array}{l}0.1032 * * * \\
(0.0276)\end{array}$ & $\begin{array}{l}0.1137 * * * \\
(0.0171)\end{array}$ \\
\hline $\mathrm{HUM}_{\mathrm{t}-1}$ & $\begin{array}{c}0.0212^{* *} \\
(0.0143)\end{array}$ & $\begin{array}{l}0.0323 * * * \\
(0.0124)\end{array}$ & $\begin{array}{l}0.0542^{* *} \\
(0.0187)\end{array}$ & $\begin{array}{l}0.0752 * * \\
(0.0436)\end{array}$ & $\begin{array}{l}0.0824 * * * \\
(0.0132)\end{array}$ & $\begin{array}{l}0.0478^{* *} \\
(0.0225)\end{array}$ \\
\hline RATIO & $\begin{array}{l}-0.2213^{*} \\
(0.1297)\end{array}$ & $\begin{array}{l}-0.2197 * * * \\
(0.0921)\end{array}$ & $\begin{array}{l}-0.2068^{* *} \\
(0.1045)\end{array}$ & $\begin{array}{l}-0.2132 * * \\
(0.1076)\end{array}$ & $\begin{array}{l}-0.2386^{* * *} \\
(0.0119)\end{array}$ & $\begin{array}{l}-0.2469^{* *} \\
(0.2121)\end{array}$ \\
\hline PERCA & $\begin{array}{c}0.3248^{* *} \\
(0.1512)\end{array}$ & $\begin{array}{c}0.4241 * * \\
(0.2131)\end{array}$ & $\begin{array}{l}0.3958^{* *} \\
(0.1923)\end{array}$ & $\begin{array}{l}0.4612^{* *} \\
(0.2242)\end{array}$ & $\begin{array}{l}0.3497 * * \\
(0.1716)\end{array}$ & $\begin{array}{l}0.3136^{* *} \\
(0.1534)\end{array}$ \\
\hline POP & $\begin{array}{l}0.3235^{* *} \\
(0.1725)\end{array}$ & $\begin{array}{l}0.3616^{* *} \\
(0.1726)\end{array}$ & $\begin{array}{l}0.3575^{* *} \\
(0.1785)\end{array}$ & $\begin{array}{l}0.4768^{* *} \\
(0.2414)\end{array}$ & $\begin{array}{l}0.3023 * * * \\
(0.1143)\end{array}$ & $\begin{array}{l}0.4298 * * \\
(0.2017)\end{array}$ \\
\hline Overall R-squared & 0.6732 & 0.6693 & 0.7032 & 0.6913 & 0.6673 & 0.7124 \\
\hline $\mathrm{P}$-value for the F-test & 0.0000 & 0.0000 & 0.0000 & 0.0000 & 0.0000 & 0.0000 \\
\hline
\end{tabular}

Notes: The *, ** and *** denote $10 \%, 5 \%$ and $1 \%$ significance levels, respectively. Standard errors are in the parentheses.

Table 4 Two-way causality between output per worker and combined level of education: Model without trade Table 4a Dependent variable: Output per worker

\begin{tabular}{|c|c|c|c|c|c|c|}
\hline \multirow{2}{*}{ Variable } & \multicolumn{2}{|c|}{ Primary+ Secondary } & \multicolumn{2}{|c|}{ Secondary+ College } & \multicolumn{2}{|c|}{ All three levels } \\
\hline & 1991-2005 & 1994-2005 & 1991-2005 & 1994-2005 & 1991-2005 & 1994-2005 \\
\hline HUM & $\begin{array}{l}0.2326^{* *} \\
(0.1216)\end{array}$ & $\begin{array}{l}0.2233^{* *} \\
(0.1103)\end{array}$ & $\begin{array}{l}0.2198 * * * \\
(0.0569)\end{array}$ & $\begin{array}{c}0.2231 * * \\
(0.1213)\end{array}$ & $\begin{array}{c}0.2624 * * \\
(1302)\end{array}$ & $\begin{array}{l}0.2398^{* * *} \\
(0.1201)\end{array}$ \\
\hline FI & $\begin{array}{l}0.2219 * * \\
(0.1201)\end{array}$ & $\begin{array}{l}0.3102^{* *} \\
(0.1523)\end{array}$ & $\begin{array}{l}0.3329 * * \\
(0.1619)\end{array}$ & $\begin{array}{l}0.3387^{* *} \\
(0.1741)\end{array}$ & $\begin{array}{l}0.3437 * * * \\
(0.1014)\end{array}$ & $\begin{array}{l}0.3298 * * \\
(0.1675)\end{array}$ \\
\hline $\mathrm{OUT}_{\mathrm{t}-1}$ & $\begin{array}{l}0.0413^{* *} \\
(0.0183)\end{array}$ & $\begin{array}{c}0.0363 * * \\
(0.0145)\end{array}$ & $\begin{array}{l}0.0356 * * * \\
(0.0153)\end{array}$ & $\begin{array}{l}0.0431^{* *} \\
(0.0221)\end{array}$ & $\begin{array}{l}0.0335 * * \\
(0.0157)\end{array}$ & $\begin{array}{l}0.0497^{* *} \\
(0.0286)\end{array}$ \\
\hline CAP & $\begin{array}{c}0.2978 * * \\
(0.1565)\end{array}$ & $\begin{array}{l}0.2735 * * * \\
(0.0386)\end{array}$ & $\begin{array}{l}0.2986 * * * \\
(0.0476)\end{array}$ & $\begin{array}{l}0.2874^{* * *} \\
(0.0329)\end{array}$ & $\begin{array}{l}0.2712^{* * * *} \\
(0.0386)\end{array}$ & $\begin{array}{l}0.2721^{* *} \\
(0.1402)\end{array}$ \\
\hline TRADE & $\begin{array}{l}0.0225^{* *} \\
(0.0121)\end{array}$ & $\begin{array}{l}0.0312 * * \\
(0.0159)\end{array}$ & $\begin{array}{l}0.0297 * * * \\
(0.0143)\end{array}$ & $\begin{array}{l}0.0226 * * \\
(0.0132)\end{array}$ & $\begin{array}{l}0.0252 * * \\
(0.0129)\end{array}$ & $\begin{array}{l}0.0228^{* *} \\
(0.0121)\end{array}$ \\
\hline FOCAP & $\begin{array}{l}0.0315^{* *} \\
(0.0142)\end{array}$ & $\begin{array}{c}0.0379 * * \\
(0.0174)\end{array}$ & $\begin{array}{l}0.0374 * * \\
(0.0184)\end{array}$ & $\begin{array}{l}0.0468^{* *} \\
(0.0205)\end{array}$ & $\begin{array}{l}0.0332 * * \\
(0.0169)\end{array}$ & $\begin{array}{l}0.0445^{* *} \\
(0.0227)\end{array}$ \\
\hline Overall R-squared & 0.6870 & 0.7852 & 0.7034 & 0.6954 & 0.7463 & 0.7645 \\
\hline Observations & 145 & 116 & 145 & 116 & 116 & 87 \\
\hline
\end{tabular}

Table 4b Dependent variable: Human capital at combined level of education

\begin{tabular}{|c|c|c|c|c|c|c|}
\hline \multirow{2}{*}{ Variable } & \multicolumn{2}{|c|}{ Primary+ Secondary } & \multicolumn{2}{|c|}{ Secondary+ College } & \multicolumn{2}{|c|}{ All three levels } \\
\hline & 1991-2005 & $1994-2005$ & 1991-2005 & $1994-2005$ & $1991-2005$ & 1994-2005 \\
\hline OUT & $\begin{array}{c}0.2343 * * \\
(0.1197)\end{array}$ & $\begin{array}{c}0.2413 * * \\
(0.1102)\end{array}$ & $\begin{array}{l}-0.0796 * * \\
(0.0332)\end{array}$ & $\begin{array}{l}-0.0823^{*} \\
(0.0313)\end{array}$ & $\begin{array}{c}0.1187 * * \\
(0.0276)\end{array}$ & $\begin{array}{c}0.1203 * * \\
(0.0171)\end{array}$ \\
\hline $\mathrm{HUM}_{\mathrm{t}-1}$ & $\begin{array}{l}0.0298 * * * \\
(0.0102)\end{array}$ & $\begin{array}{l}0.0286^{* *} \\
(0.0132)\end{array}$ & $\begin{array}{l}0.0567 * * * \\
(0.0124)\end{array}$ & $\begin{array}{l}0.0764^{* *} \\
(0.0402)\end{array}$ & $\begin{array}{l}0.0675^{* * *} \\
(0.0128)\end{array}$ & $\begin{array}{l}0.0412^{* *} \\
(0.0213)\end{array}$ \\
\hline RATIO & $\begin{array}{l}-0.2132 * \\
(0.1224)\end{array}$ & $\begin{array}{l}-0.0259 * * * \\
(0.0984)\end{array}$ & $\begin{array}{l}-0.2186^{* *} \\
(0.1012)\end{array}$ & $\begin{array}{l}-0.2265^{* *} \\
(0.1024)\end{array}$ & $\begin{array}{l}-0.2154^{* *} \\
(0.1043)\end{array}$ & $\begin{array}{l}-0.2246^{* * *} \\
(0.0191)\end{array}$ \\
\hline PERCA & $\begin{array}{c}0.3925^{* *} \\
(0.1943)\end{array}$ & $\begin{array}{l}0.3647 * * * \\
(0.2130)\end{array}$ & $\begin{array}{l}0.4023^{* *} \\
(0.1989)\end{array}$ & $\begin{array}{l}0.4034^{* *} \\
(0.2132)\end{array}$ & $\begin{array}{l}0.3924 * * \\
(0.1919)\end{array}$ & $\begin{array}{l}0.3634^{* * *} \\
(0.1032)\end{array}$ \\
\hline POP & $\begin{array}{l}0.4021^{* * *} \\
(0.1231)\end{array}$ & $\begin{array}{l}0.3369 * * \\
(0.1674)\end{array}$ & $\begin{array}{l}0.3463^{* *} \\
(0.1703)\end{array}$ & $\begin{array}{l}0.4024^{* *} \\
(0.2034)\end{array}$ & $\begin{array}{l}0.3745^{* * *} \\
(0.1042)\end{array}$ & $\begin{array}{l}0.4312^{* *} \\
(0.2133)\end{array}$ \\
\hline Overall R-squared & 0.6925 & 0.7214 & 0.6846 & 0.6794 & 0.7043 & 0.6714 \\
\hline P-value for the F-test & 0.0000 & 0.0000 & 0.0000 & 0.0000 & 0.0000 & 0.0000 \\
\hline
\end{tabular}

Notes: The *,** and *** denote $10 \%, 5 \%$ and $1 \%$ significance levels, respectively. Standard errors are in the parentheses. 
We then investigate the combined effects of two or three levels of education. Table 3a and Table 3b report the results for the first and the second equations in system (3), respectively, for the model with trade. They show that the combined effect of primary and secondary graduates, secondary and college graduates, and that of all three levels of graduates are all highly significant.

Concerning the effects of output per worker on combined levels of education, we again find that the results are mixed. The effects on the combined level of primary and secondary education, as well as on a combination of all three levels, are positive and significant. However, that on the combined level of primary and secondary education is double that of all three levels. The effect of output per worker on the combined level of secondary and college education is negative and significant. The results are robust to alternative sample periods of 1991-2005 and 1994-2005. Results for the model without trade, which are shown in Table 4, are also similar to those in Table 3.

The results for the effect of output per worker on human capital accumulation in all above discussed tables, call for a combination of explanations in Kumar (2003) and Vu and Hammes (2007). On one hand, education increases productivity that increases per capita income. This increase in per capita income in turn increases school enrollments and graduates since more people can afford education as argued in Vu and Hammes (2007). On the other hand, the increase in per capita income increases the opportunity cost of going back to schools. People might prefer working to going back to school, where they have to sacrifice their income. Therefore increase in productivity decreases school enrollments and graduates as argued in Kumar (2003). Hence, the combined effects might be positive and highly significant with different magnitudes or negative.

Nonetheless, the negative effect of output per worker on human capital accumulation is the evidence only at college level or a combination of college and secondary school levels in our paper instead of the negative effect on secondary school level alone as shown in Kumar (2003). We believe that our case makes more sense, as the opportunity cost of going to colleges is much higher than that of going to secondary schools. This is especially true for the case of China, where college enrollments are limited to a small percentage of the population.

\section{Conclusion}

Economic growth theory posits that investment in human capital improves output per worker. However, empirical results, using single equation estimation for China, have been inconclusive and depend on levels of education. In this paper, VAR estimations are performed to control for the two-way causality. Macroeconomic data are averaged every three-year to reduce fluctuation effect of the business cycle in the short run. Numbers of graduates at each level of education are divided by numbers of workers to use as a proxy for investment in human capital that contributes to changes in real GDP per worker in China.

The results show that investment in human capital is profitable in the sense that it increases output per worker at all three levels of education. Additionally, the combined effects of secondary and primary graduates, secondary and college graduates, as well as those of all three levels of education are all highly significant.

However, the effect of output per worker on each level of education and combined levels of education are mixed. The effects are positive and highly significant effect for primary and secondary education, but the effect on primary education is more than triple that on secondary education. The effect is negative and statistically significant for college education. The effects on the combined level of primary and secondary education, as well as on a combination of all three levels, are positive and significant. Again, that on the combined level of primary 
and secondary education is double that of all three levels. The effect of output per worker on the combined level of secondary and college education is negative and significant.

Our results are robust to sample size changes and alternative model specifications. They are also similar to the results obtained from microeconomic data and reconfirm the importance of investment in human capital on output per worker in China. In the case of primary education, it is more so in the indirect role of this level to provide the necessary prerequisites to enroll in higher level of education. Since our paper focuses on human capital and output per worker, the question of a possible two-way causality between school enrollments and total factor productivity is left for future research.

\section{References:}

Arellano, M. \& S. Bond. (1991). Some test of specification for panel data: Monte Carlo evidence and an application for employment equations. The Review of Economic Studies, 58(2), 277-297.

Barro, R. \& X. Sala-i-Martin. (2004). Economic growth. MA dissertation, Massachusetts Institute of Technology.

Chen, B. \& Y. Feng. (2000). Determinant of economic growth in China: Private enterprise, education, and openness. China Economic Review, 11(1), 1-15.

Demuger, S.. (2001). Infrastructure and economic growth: An explanation for regional disparities in China. Journal of Comparative Economics, 29(1), 95-117.

Fleisher, B.. (2002). Higher education in China, a growth paradox?. Department of Economic, Ohio State University, 1-23, mimeo.

Fleisher, B., Hu. Y. \& L. Haizheng. (2006). Economic transition, higher education, and worker productivity in China. Working paper (submitted), Department of Economic, Ohio State University.

Kennedy, P.. (2003). A guide to econometrics. Cambridge: The MIT Press.

Kumar, K. B.. (2003). Education and technology adoption in a small open economy: Theory and evidence. Macroeconomic Dynamic, 7(4), 586-617.

Hua, P.. (2006). How education influences productivity growth? Evidence from the Chinese provinces. Working paper, CERDI-INDIRECT, CNRS-University d' Auvergne.

Pindyck, R. S. \& D. Rubinfeld. (1998). Econometric models and economic forecast (4th ed.). Boston: Mc Graw-Hill/Irwin.

Thirwall, A. P.. (2006). Growth \& development with special references to developing economies. New York: Palgrave MacMillan.

Vu, T. B. \& D. Hammes. (2007). Education and productivity: A fixed effect three stage least squares approach. The 10th International Conference of the Society on Business and Economic Development. Kyoto.

Vu, T.B. \& Im, E.. (2007). Education and productivity in China: New approach, new results. The 41st Asian Studies on the Pacific Annual Conference. Honolulu.

(Edited by Ruby and Gracie) 